SAND 91-1168

DE92 000608

Unlimited Release

Printed 1991

\title{
The Implementation of a Standards Based Heterogeneous Network
}

\author{
John M. Eldridge and Lawrence F. Tolendino \\ Sandia National Laboratories \\ Division 1934 \\ Albuquerque, NM 87185
}

August 5, 1991

\begin{abstract}
Computer networks, supporting an organization's activities, are prevalent and very important to the organization's mission. Implementing a heterogenous organizational network allows the staff to select the computing environment that best supports their job requirements. This paper outlines the lessons learned implementing a heterogenous computer network based on networking standards such as TCP/IP and Ethernet. Such a network is a viable alternative to a proprietary, vendor supported network and can provide all the functionality customers expect in a computer network.
\end{abstract}

\section{MASTER}




\section{Trademarks}

Apollo and Domain are registered trademarks of Hewlett-Packard Company.

Ethernet is a trademark of Xerox Corporation.

IBM, IBM PC, IBM AT are registered trademarks, and PC/XT and DOS are trademarks of International Business Machines Corporation.

INGRES is a trademark of ASK Computer Systems, Inc.

INTEL is a registered trademark of Intel Corporation.

MS-DOS and Microsoft are registered trademarks of Microsoft Corporation in the U.S.A. and other countries.

NFS is a trademark of Sun Microsystems, Inc.

DEC, VAX, VMS, and DECnet are trademarks of Digital Equipment Corporation.

$3 \mathrm{COM}$ is a trademark of $3 \mathrm{COM}$ Corporation.

PC/TCP and FTP Software are registered trademarks of FTP Software, Inc.

PostScript is a trademark of Adobe Systems, Inc.

UNIX is a registered trademark of AT\&T in the U.S.A and other countries. 


\section{Table of Contents}

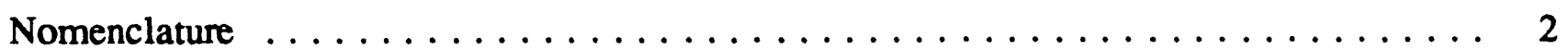

Management Summary $\ldots \ldots \ldots \ldots \ldots \ldots \ldots \ldots \ldots \ldots \ldots \ldots \ldots$

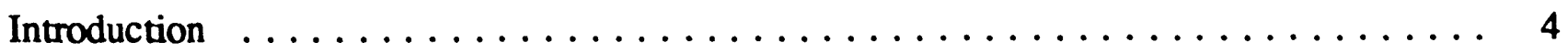

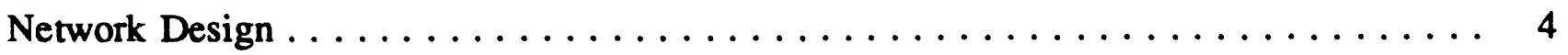

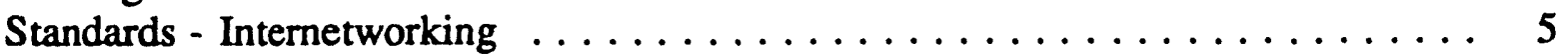

Physical Interconnection Media $\ldots \ldots \ldots \ldots \ldots \ldots \ldots \ldots \ldots \ldots$

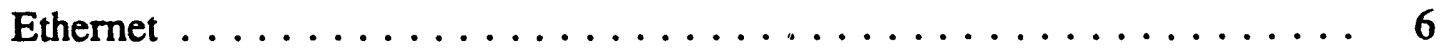

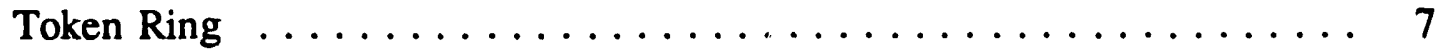

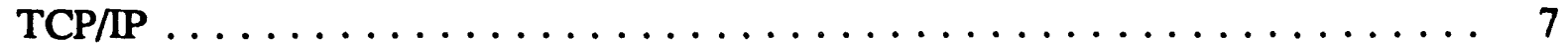

Network Configuration $\ldots \ldots \ldots \ldots \ldots \ldots \ldots \ldots \ldots \ldots \ldots \ldots, 8$

Network Services $\ldots \ldots \ldots \ldots \ldots \ldots \ldots \ldots \ldots \ldots \ldots \ldots$

Network File Server $\ldots \ldots \ldots \ldots \ldots \ldots \ldots \ldots \ldots \ldots, 8$

Network Printers and Output $\ldots \ldots \ldots \ldots \ldots \ldots \ldots \ldots \ldots$

Backup TAR, rsh etc. $\ldots \ldots \ldots \ldots \ldots \ldots \ldots \ldots \ldots \ldots \ldots$

Network Details . ........................... 9

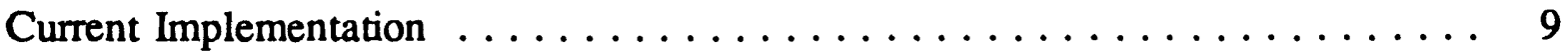

Growth Path and Design Characteristics .................. 10

Maintenance and Operation ...................... 11

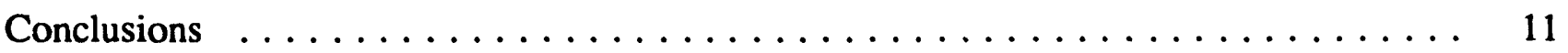

Deciding on Use of a Heterogeneous Network $\ldots \ldots \ldots \ldots \ldots \ldots \ldots \ldots 11$

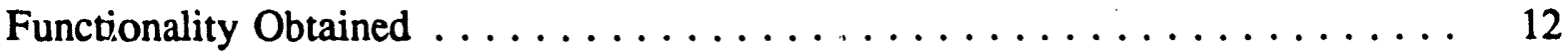

Advantages and Disadvantages $\ldots \ldots \ldots \ldots \ldots \ldots \ldots \ldots \ldots \ldots \ldots \ldots$

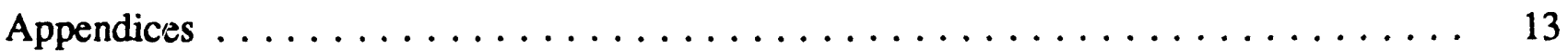

A.ppendix A - Platforms and Descriptions of Products Used .......... 13

Appendix B - SNL Networking Environments $\ldots \ldots \ldots \ldots \ldots \ldots \ldots \ldots$ 


\section{Nomenclature}

DEC Digital Equipment Corporation

FTP

File Transfer Protocol

ISO

International Standards Organization

LAN Local Area Network

NFS Network File System

NSFnet National Science Foundation Network

OSI Open Systems Interconnection

PBX Private Branch Exchange

RAN Restricted Access Network

SMTP Simple Mail Transfer Protocol

SSN Secure Supercomputing Network

SCN Secure Computing Network

TCP/IP Transmission Control Protocol/Internet Protocol suite

VMS Virtual Memory System

WBCN Wide Band Communications Network 


\section{Management Summary}

Division 1934, the Computer Communications Design Division, has worked on computer networking projects for more than ten years. During that period an organizational network grew composed of a heterogeneous mix of computing platforms ranging from DEC VAX time sharing systems to PCs and workstations. However, a proprietary network architecture and associated protocols dominated the network. This situation did not provide an environment that encouraged the sharing of data and devices. There were two options to improve the situation. The heterogeneous network could be replaced with a homogeneous one or standard networking architectures and protocols could be used to support the existing mix of computing platforms and operating systems.

Standards are referred to throughout this paper. We have used standard to indicate objects or concepts that are widely accepted, used, and supported by a large number of individuals, organizations, and vendors. Standards described in this paper are not necessarily specifications which have been formally approved by a national or international organization ${ }^{1}$.

The staff decided to use networking standards. This allowed the staff to choose the computing platform that best supported their job requirements while still being a part of the organizational network. The organizational network uses Ethernet and the TCP/IP protocol suite. A network server node is part of the network. It runs UNIX System V and has a variety of output devices, a generous amount of disk storage, and other specialized peripherals. Using standards has allowed this network to be developed for a very modest cost while providing network access to all the members of the organization. Using industry standards has allowed the purchase of hardware and software at commodity prices instead of the higher prices associated with the proprietary components of one vendor. In addition, the organization will not be held hostage by any vendor nor will the network depend on the fortunes of any vendor.

While supporting a heterogeneous network based on recognized standards may not be the best network answer for all organizations, we believe that the advantages of installing such a network far out weigh any disadvantages. In reporting the design of this working heterogeneous network, other groups at Sandia National Laboratories (SNL) may benefit from the experiences learned.

${ }^{1}$ Two widely recognized organizations that formally adopt and specify communication and network standards include the Institute of Electrical and Electronic Engincers (IEEE) and the International Telegraph and Telephone Consultative Committee (CCITT). 


\section{Introduction}

Division 1934, the Computer Communications Design Division, and Division 1935, the Communications Services Division, have the responsibility to evaluate, develop, implement, and maintain data communication services for Sandia National Laboratories, Albuquerque. The staff has relied on a variety of computing platforns to fulfill the division's mission. These computers range from personal computers to UNIX based workstations and multiuser Digital Equipment Corporation VAX time sharing systems. The staff used whichever computing platform best suited their current task.

Over time this mode of operation resulted in several "islands" of computing. Such an environment inhibited cooperative projects among the staff and led to a duplication of hardware such as printers. The staff recognized this less than ideal environment as a problem for current operations and a future that promised reduced funding.

The staff gradually formed a consensus that networking these islands would provide operational and budgetary benefits if the networking could meet certain requirements. First the staff had to be free to continue to use the competitive platforms and operating systems that best suited their needs. Second, the resulting network should function within defacto industry standards such as Ethernet and TCP/IP. By operating within standards, the division gained the most opportunity for industry support and growth. The division recognized that such a network would be a challenge to build because it would not be the "ideal" network for many computing platforms. It might impose some restrictions on the system's functionality. However, the staff believed that if they exercised sufficient self discipline a network could be created that would provide adequate functionality and that would span the wide variety of computing platforms and operating systems installed.

\section{Network Design}

When the division began to build its existing heterogeneous network, it already had many Apollo workstations. These workstations were running a proprietary multiuser and multitasking operating system and were networked with proprietary Apollo token ring facilities. This Apollo network used a sophisticated network operating system and provided a very high level of network integration and functionality. The Apollo workstations provided a good base for developing communication systems control code and for other development tasks. However, some staff found that the Apollo environment was not capable or not suited for their project tasks. Some staff were using simpler and easier to use personal computers that provided a wide range of productivity software. Other staff were using a mix of specialized workstations necessary for their projects. Generally, all staff had asynchronous terminal access to a Digital Equipment Corporation VAX 8350 computer. The staff used this computer to develop and maintain the Tech Control Center communications database and for general computing. Additionally, each staff member typically had a printer and a variety of other peripherals directly attached to their computers. 
Project team members found it desirable to be able to share electronic word processing documents and other data as they worked on different project tasks. Also, the division members desired to be able to take advantage of high performance plotters and color printers directly attached to other division member's computers. To address these desires the division set a goal of integrating these various computing platforms, output devices, and peripherals into a single network. The division decided to base the network on widely accepted industry standards to achieve this goal of a single integrated network. The staff chose standards for both the physical communications medium and the neiworking protocols in order to support the widest variety of computing platforms.

\section{Standards - Internetworking}

The use of standard networking hardware and software formed the basis for our heterogeneous networking effort. The core or hub of the network, that part of the network that integrated the computing "islands" into a single network, uses defacto internetworking standards. The defacto standards that existed and the installed wire plant encouraged the use of Ethernet communications hardware using twisted pair or fiber optic cables. The choice of a higher level network protocol was equally obvious. With the trend toward UNIX and nonproprietary systems, the TCP/IP protocol suite is available for all the platforms within the organization. The protocol suite is even available for personal computers and DEC VAX computers running VMS. This heterogeneous network derives its functionality from these "standard" network protocols and network communication interfaces.

Given the background and experience of the staff, it was natural to think of networking in terms of a layered network model. One such model is the International Standards Organization (ISO) Open System Interconnection (OSI) model. The OSI model describes computer networks in terms of a hierarchy of networking protocols. Each protocol or layer performs a specific set of functions and has a fixed communication interface to the layer above and below in the hierarchy. The lowest layer in the hierarchy is the layer that controls the computer's network hardware. The top most layer presents data to and receives commands from the user.

The TCP/IP Internet Protocol Suite, commonly called TCP/IP, is an example of a layered network model. TCP/IP, developed through research funded by the Defense Advanced Research Projects Agency, does not specifically implement all the layers described in the OSI model. TCP/IP, however, does implement the functionality of the model. The TCP/AP protocol suite includes the communication protocols for reliable computer to computer data exchange and commonly used application programs which use the data exchange protocol. The common application programs provide much of the functionality to perform useful network tasks. Figure 1 diagrams the layers of the OSI model and the TCP/IP protocol suite. The physical and link layers of the OSI model deal with the communication equipment that interconnects computers and the electrical signaling required for communications over that equipment. The transport level protocol provides a systematic procedure or method for two 
or more computers to exchange data reliabiy. Network applications that use the transport level protocol provide the functionality and utility desired in the network.

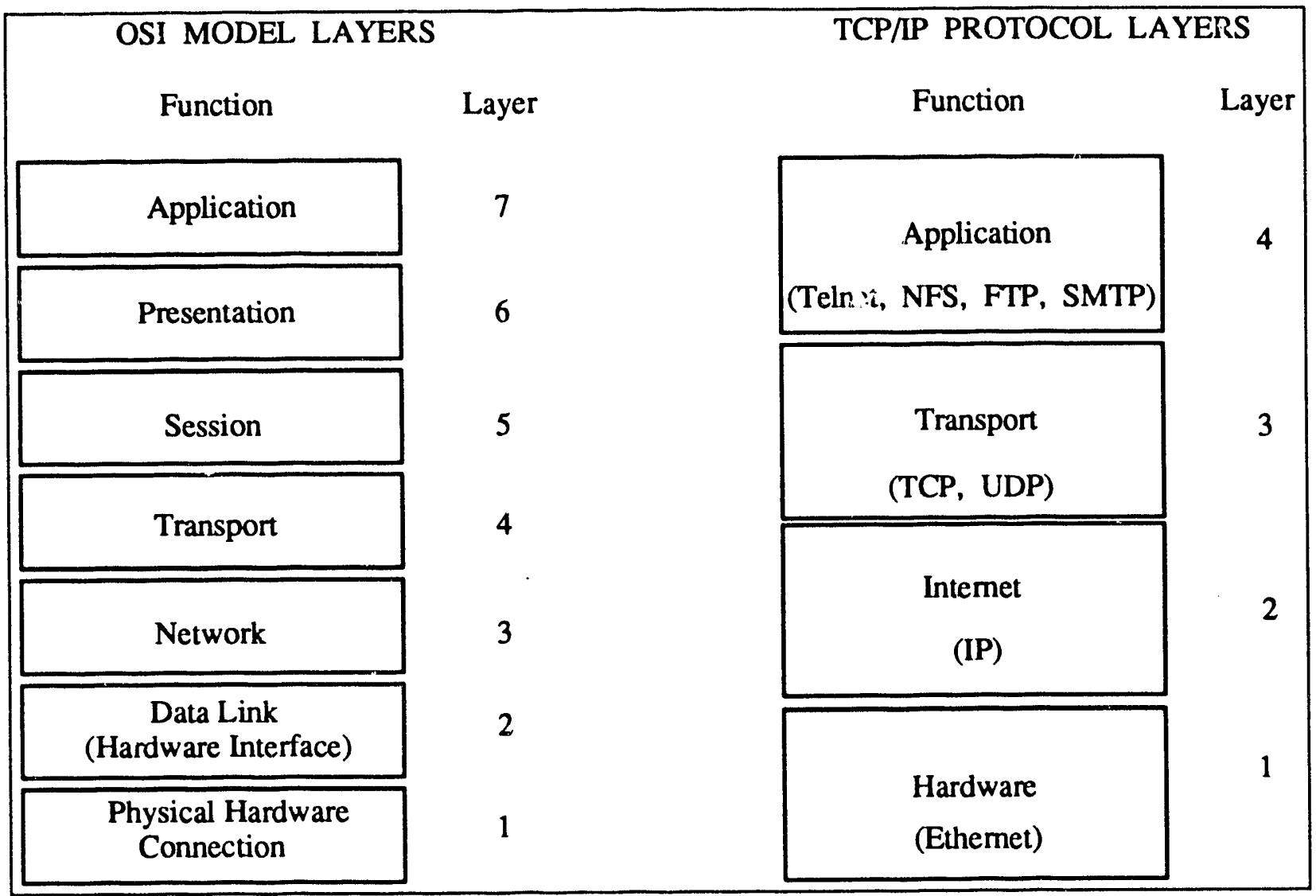

Figure 1

\section{Physical Interconnection Media}

\section{Ethernet}

Ethernet interface cards are available for all the computing platforms installed or projected for future installation. Given the universal availability of Ethernet, Ethernet became the defacto interconnection standard ${ }^{2}$. Ethernet did not replace proprietary

\footnotetext{
${ }^{2}$ While ethernet controllers for most computers support both an Attachment Unit Interface (AUI) 15 pin connector and a radio frequency BNC type connector, the division relied exclusively on the AUI. The AUI is described in the IEEE 802.3 specification. The major difference between the two interfaces is that the AUI requires an external transceiver to connect to the ethemet coaxial cable (or as more likely a piece of communication equipment that emulates the traditional ethernet coaxial cable). In the BNC interface, the transceiver is an integral component of the controller. While in some cases there are up front cost advantages to using the BNC interface, there are several overriding disadvantages. The majority of commercial equipment for implementing, managing, and extending an ethernet rely on the AUI. Additionally, equipment that implements the AUI is readily available that can use fiber optic cable and twisted wire pairs for the transmission medium in
} 
networking interfaces such as the Apollo token passing ring. But over time, it became a very important adjunct with certain workstations containing both proprietary interfaces and Ethernet interfaces. These nodes, such as the Apollo workstation server, became gateways between the proprietary worlds of the Apollo token passing ring and the heterogeneous world of Ethernet. Indeed, as the organizational network evolved the importance of the proprietary ring network declined while the importance of the Ethernet increased. This evolution reached the point that Apollo workstations used Ethernet as the primary network interface. The ease of configuring and supporting a "standard" interface outweighed the possible performance advantages of the proprietary network ring interfaces.

\section{Token Ring}

Token ring local area networks are represented in the organizational network by a 12 Mbit HP/Apollo ring. This proprietary ring uses coaxial cable and the staff has developed interfaces to implement the ring on fiber optic cable. The division does not use IBM style token ring networks.

\section{TCP/IP}

In developing this network, the staff desired several network functions and capabilities. These capabilities included the ability of a division member to login remotely to other computers on the network, the ability to move data between computers on the network, and the ability to share common resources among users of the network. The TCP/IP protocol suite provides a range of utilities to obtain this network functionality.

Telnet - $\quad$ Telnet is a TCP/IP suite application program which emulates a rernote terminal. Telnet establishes a communication channel between an interactive process on a remote computer and the keyboard and display on the user's local computer.

FTP - $\quad$ FTP is the TCP/IP file transfer protocol that implements file transfer between computer systems. The protocol has a long development history and is available for computers ranging from CRAY supercomputers to desktop PCs.

NFS - $\quad$ NFS is the network file system protocol, pioneered by SUN Microsystems Inc., that has become a defacto standard for network file system access. Remote file systems on any host running an NFS server may be accessed locally as if the remote file system was an integral part of the local computer. NFS is available on a variety of computers and is considered a common TCP/IP application.

addition to coaxial cable. 
SMTP - $\quad$ SMTP is the Simple Mail Transfer Protocol. One uses it to send and receive electronic mail and store received messages.

Ipr - $\quad$ Remote line printing utility facilitates file printing across the network to a server with attached printers or to printers directly attached to the network.

\section{Network Configuration}

Large segments of this heterogeneous network use standard interfaces and protocols, however, other segments of the network still use proprietary and non-standard network interfaces and protocols. The network core or central part of the network uses ethernet and TCP/IP. Gateway computers attached to the standards based network core integrate the non-standard network segments into the network. The gateways perform both signal and protocol conversion. Electrical signal conversion between ethernet and token ring and TCP/IP, DECnet, and DEC's LAT protocol conversion is necessary. New machines added to network use Ethernet and TCP/IP and are attached directly to the network core.

An important component of the network is a dedicated computer or server that performs common network tasks. All members of the organization use this machine. Currently, the division uses an Apollo workstation running UNIX for the server. The server requires a multiuser and multitasking operating system to accommodate the variety of tasks that the server needs to perform and to service the multiple network users. The server also provides routing and gateway connections to other networks.

\section{Network Services}

\section{Network File Server}

The network file server has become an indispensable part of the organization LAN. It provides a means of sharing files between system nodes and a convenient location for backing up user files. With the network file server easily accessible through the FTP and NFS protocols, network users are able to produce file backups easily. Indeed, with the network server's file system mounted on a user's node, it is as easy to create a file system backup on the server as it is to create one on a directly connected disk drive. Staff members install software packages for all machines in the network on the server. This allows easy network installation of these packages on new nodes added to the network. Staff load software from tape or floppy disk and configure the software on the server once. They do not then need to reload and configure the software for each new computer installation. The staff runs infrequently used software packages directly from the server. Therefore, the organization needs fewer licenses and less disk space. 
Providing network printers and other output devices to the entire network as a network resource instead of directly connecting them to each node has made more sophisticated and capable devices available. Both black-and-white and color PostScript printers are available to network users. These printers have been invaluable for providing graphic aids and desktop publishing support. In addition, a Hewlett-Packard DraftPro plotter is available that can provide a variety of plot sizes.

Backup TAR, rsh etc.

Along with the usual network services of data and device sharing, a network based on standards such as UNIX can provide unique services such as TAR, rsh, etcetera. A service such as TAR, a tape archiving utility, can provide users access to data written on any system in the world. RSH, the remote shell facility, allows network users to submit system level commands to a UNIX based server. These services and others provide an added dimension to the network and are available because the network is designed with recognized standards.

\section{Network Details}

\section{Current Implementation}

The current network implementation is a heterogeneous network of Pcs, workstations, and time sharing computer systems. The network includes workstations manufactured by HP/Apollo and SUN, PCs from IBM, PC clones from a variety of manufacturers, Apple computers, and DEC VAX time share systems. The network has both an Ethernet component and an Apollo token ring component. The staff implemented the Ethernet with Synoptic equipment within our lab area and PBX LANmark circuits elsewhere. Current trends favor the growth of the Ethernet component at the expense of the ring. An Apollo workstation functions as a network server for file storage and printing. The Apollo workstation also functions as a network interface for a small group of personal computers, since these PCs do not implement a standard network protocol. Those PCs use proprietary software for communication with the Apollo workstation functioning as a gateway into the world of standard data communications. Figure 2 is a diagram of the network in October 1990.

Most PCs and workstations in the organization have implemented a version of TCP/IP for network communications on the local Ethernet. These communication software packages come from a variety of manufacturers includirg HP/Apollo, Apple, SUN, FTP Software, Novell, etcetera. The staff has also installed TC.P/IP on the organization's VAX 8350 where the protocol shares an Ethernet interface with DEC's proprietary DECnet protocol. 


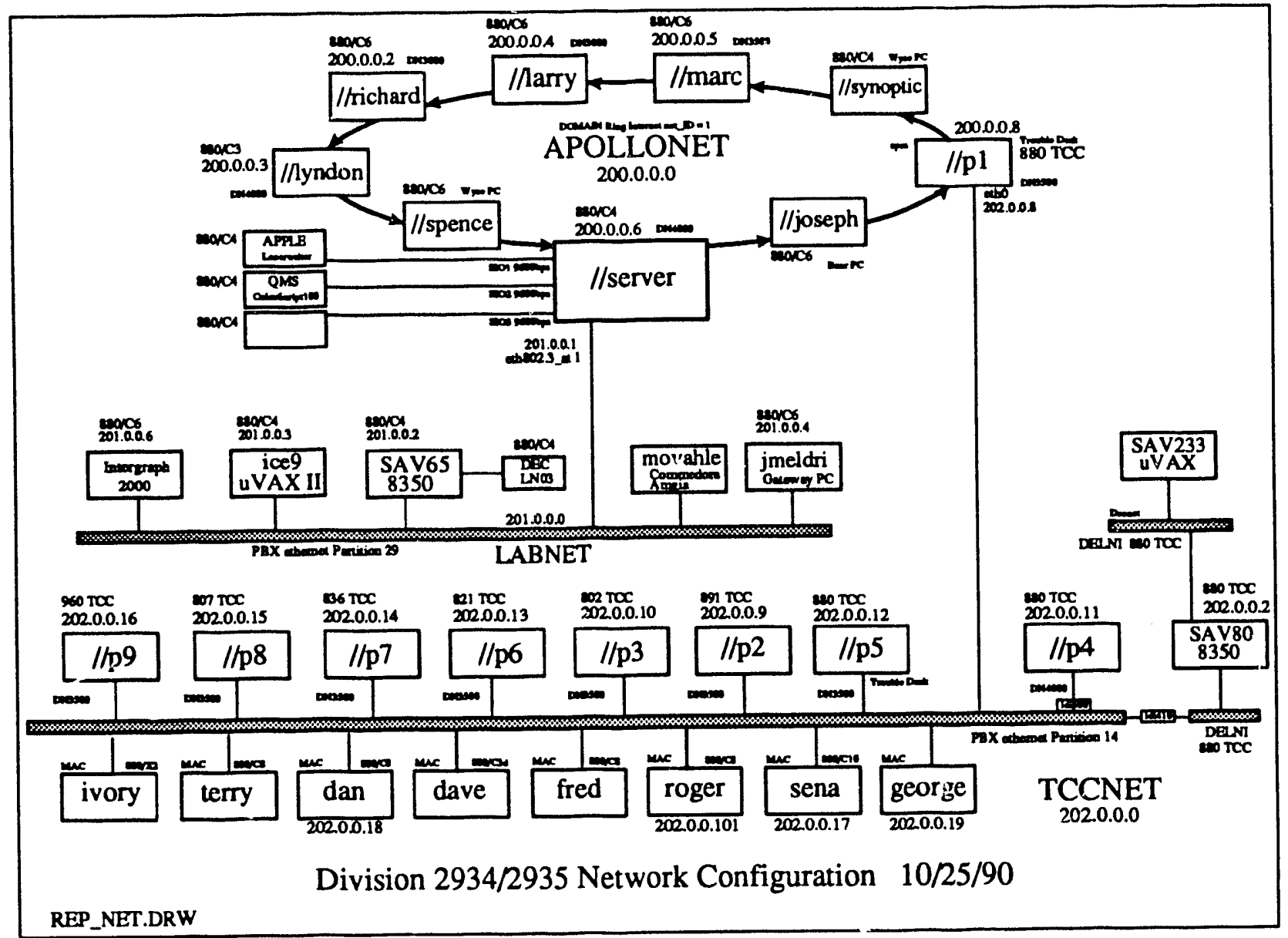

Figure 2

\section{Growth Path and Design Characteristics}

In keeping with the philosophy of using industry standards, the staff has plans to phase out the Apollo server node and replace it with a generic platform. This platform will be based on the Intel 386/486 processor running a version of industry standard UNIX (Santa Cruz Operation System V UNIX). This upgrade will require the replacement of proprietary communications software on sorne workstations and PCs in the organization with implementations of TCP/IP and NFS.

Once the new server node is operational, the staff plans to add new services. In particular, a very capable CD-ROM drive will be installed to provide CD-ROM services to all network users. Additional disk storage will be added to the server that will ni:sre than double the storage available to network users. Using a standard hardware platform with a standard operating system makes it easier to add new peripherals and capabilities as they become generally available. 


\section{Maintenance and Operation}

Maintenance of this heterogeneous network becomes an organizational responsibility. Therefore, it is imperative that accurate records are available to facilitate network trouble shooting. In such a network implementation, one or more organization personnel must have responsibility for network performance. All personnel must respect the rights of others wr.en making changes to their configurations. The network manager or managers are respons'sle for the orderly integration of the various network nodes. The network manager must assign TCP/IP addresses and install appropriate network interconnections.

Maintenance of the individual network nodes is most commonly the vendor's responsibility. However more generic PCs are joining the network and maintenance of those nodes will be the user's responsibility.

Individuals responsible for implementing any network should consider the intended use and growth potential of the network and the possible interconnection to other networks. An evaluation should be made to determine which of the four SNL computing environments best matches the network. The four environments (see Appendix B) characterize networks based on network security and interconnectivity to organizations outside SNL. These computing environments are not specific networks, however there are large existing networks within each con.puting environment. SNL has acquired blocks of IP address for each of these environments from the internet community. All TCP/IP networks at SNL should use IP addresses out these available blocks. Through the use of these IP address blocks, unique addresses may be assigned to all nodes at Sandia. This becomes particularly important as network managers attempt to interconnect small isolated LANs. Likewise, network managers should carefully consider the assignment of node names, usernames, and user identifiers for a local network.

\section{Conclusions}

\section{Deciding on Use of a Heterogeneous Network}

Installing a brterogeneous network may not be the ideal solution for many organizations. The decision tr install such a network should be based on the requirements of the members of the organization AND the willingness and ability of the organization to accept network responsibility. Experience has shown that network maintenance is not a trivial task. On the contrary, network maintenance requires considerable resources. For those organizations unwilling or unable to accept network responsibilities the best alternative is a homogeneous network with the vendor assuming all responsibility. 


\section{Functionality Obtained}

Installing a standards based heterogeneous network has provided file and device sharing to all the nodes in the organizational network. More than 600 Mbytes of disk storage will soon be available to network users as well as access to sophisticated output devices. The output devices incluce both black-and-white and color PostScript printers and a large HewlettPackard plotter. Such output devices would have been prohibitively expensive for a single workstation or PC, but are very reasonable when used for an entire network. While all the expected network functionality is available, network users have been able to use the computing platform that best meets their job requirements.

\section{Advantages and Disadvantages}

The advantages of installing a heterogeneous network based on industry standards are many. The most important is the ability to communicate and interact with the widest possible group of hardware and software products.

In addition, one can be assured that one's network will not become an "orphan" if a vendor changes direction. Nor will one face the possibility of being held "hostage" by a vendor of proprietary software and hardware products. On the contrary, if one vendor's products are inadequate or too expensive there will always be an alternative.

Future growth paths are available when one follows industry standards.

There are price advantages to implementing a standards based heterogeneous network due to competition. 


\section{Appendices}

\section{Appendix A - Platforms and Descriptions of Products Used}

We use a range of computing platforms, peripheral equipment, and software on our network. We have listed the products used and found to work in this appendix. The different computer categories and architectures are listed. This list in not a catalog of all available products, merely those products tried and found to work.

\section{Mini-computers}

Digital Equipment Corporation VAX 8350 mini computer.

Operating System: $\quad$ DEC VMS, version 5.3

Network Software: $\quad$ DEC DECnet, version VAX V5.3

TCP/IP Novell Multinet version 2.1

Network Interface Cards: DEUNA

NOTE: The staff also had a MicroVax II and a VAX 11/750 running Digital's Ultrix operating system in the network. DEC bundles TCP/IP with the Ultrix operating system. The MicroVax and the VAX $11 / 750$ used DEQUNA and DEUNA Ethernet interfaces respectively.

\section{Scientific Workstations}

Scientific workstations are specialized computers suited to numerical computation, fast graphical displays, and networking. Generally, they use the UNIX System V or the Berkeley Standard Distribution operating system. TCP/IP and Ethernet are typically built into this class of computer by the manufacturer. An exception is the Apollo workstations. They use the HP/Apollo Domain operating system and may use a proprietary token passing ring network interface. Platforms from Hewlett-Packard, Digital Equipment Corporation, Apollo, and Sun Micro System all worked in the network.

\section{Personal Computers}

Division members extensively use personal computers based on the Intel $80 \times 86$ line of microprocessors running the Microsoft MS-DOS operating system. These computers are attractive because of the selection and functionality of productivity software available. Most of the division's personal computers use the Intel 80386 and 80486 microprocessors, however PC-XT and PC-AT class machines are also used. 
Operating Systems Used:

Memory Management Software Used:

- Quartcrdeck Office Systems QEMM-386

expanded memory manager, version 5.1

Networking Software Used:

- FTP Software Inc. PC/TCP Network Software for DOS, version 2.05

- HP/Apollo network operating system, version SR10.3

Network Interface Cards Used: - 3COM Inc. 3C503 and 3C505 Etherlink Plus Ethernet interface cards

- Apollo token ring interface card

NOTE: Staff members have also used the Santa Cruz Operation (SCO) UNIX System V Open Desktop product on 80386 and 80486 machines. This product includes the UNIX operating system software, a TCP/IP network package with NFS, an MS-DOS emulation package, $\mathrm{X}$-Windows graphical user interface, and INGRES database software. The 3COM 3C503 and 3C501 Ethernet cards are compatible with the SCO package.

\section{Apple McIntosh}

Operating Systems Used:

Network Software Used:

Network Interface Cards Used:
- Apple computer operating system 6.0.5

- NCSA Software Development Telnet version 2.3

- Apple computer EtherTalk Ethernet board

\section{Commodore Amiga}

Some staff in the division also use Commodore Amiga computers. The Amiga is a capable multimedia computing platform. Video and advanced audio capabilities are standard features on all Amiga computers. Staff members have successfully integrated the Amiga 2000 into the division network.

Operating System Used:

Network Software Used:

Network Interface Cards Used: - A2065 Ethernet interface
- Commodore Amiga Workbench version 1.3

- Commodore Amiga AS 225 TCP/IP Networking software, release 1.0 


\section{Appendix B - SNL Networking Environments}

There are four computer and networking environments defined at Sandia National

Laboratories. These four environments, 1) External Secure Network environment, 2) Internal Secure Network environment, 3) Internal Restricted Network environment, and 4) External Open Network environment, describe computer operations and network installations based network security protections. These network environments characterize network security rather than describe specific networks. Several unrelated networks may belong to each of these environments.

The secure network environments (ESN and ISN) are characterized by only allowing access to DOE Q-cleared users, by using protected distribution system wiring, and by the potential that all computers may process or may be exposed to classified data. The open network environments (IRN and EON) are characterized by the absence of requirements for users to be DOE or DOD cleared, by the requirement to use open wiring that is disjoint from protected distribution system wiring, and by the restriction against any classified data

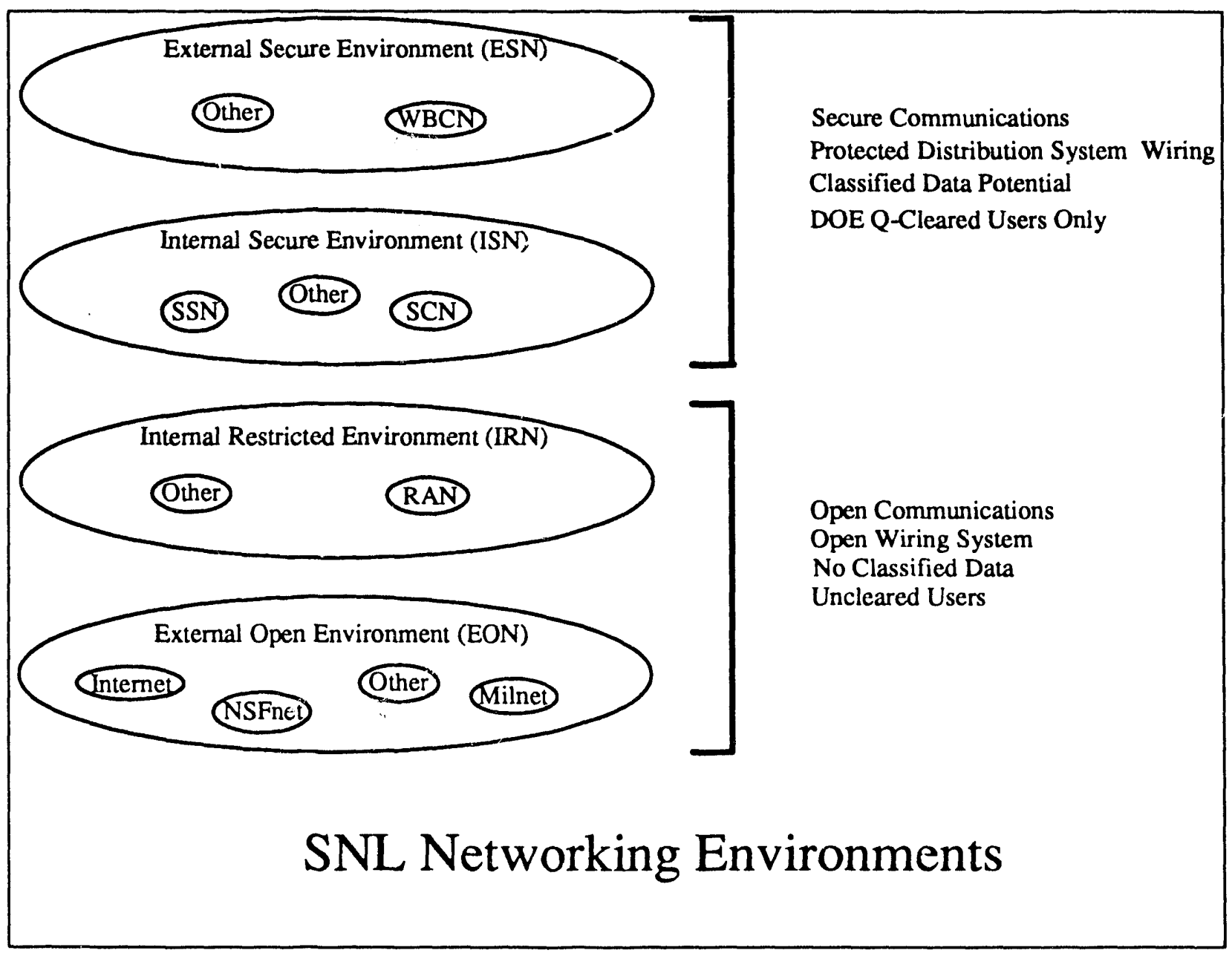


processing. The internal environments (ISN and IRN) only allow access to network and computer users that are SNL employees, SNL contractors, or other individuals directly authorized by SNL. Computer and network users in the external environments (ESN and EON) are not directly authorized access by SNL. If it is necessary, SNL may have limited authority to isolate these users from SNL computing resources in the external environments. The attached figure outlines the four networking environments including some specific networks within each environment.

SNL networks can be described in terms of the base characterizations mentioned above. However, the security requirements of specific networks may be more restrictive than the minimum set of requirements described by the four networking environments. Specific networks may impose additional restrictions and responsibilities including security plan documentation, access requirements, user requirements, and possibly other network specific requirements. 

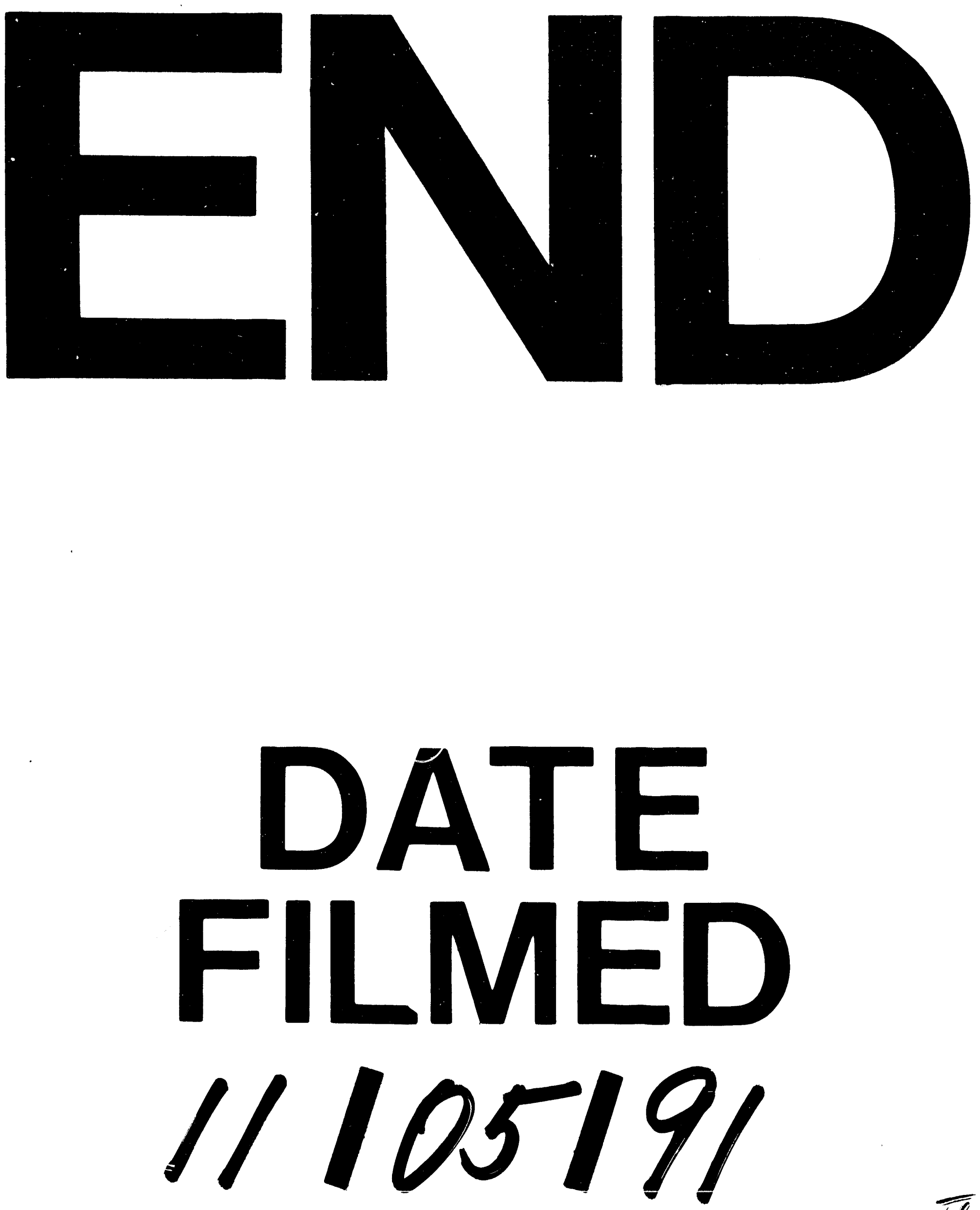


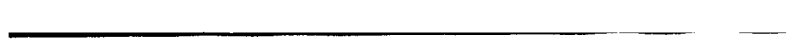

